Original Research Article

\title{
Critical analysis of drug promotional literature available to the medical practitioners
}

\author{
Kalyani V. Pai Kakode*, Padma N. Bhandare
}

Department of Pharmacology, Goa Medical College, Bambolim-Goa, India

Received: 04 February 2019

Revised: 21 March 2019

Accepted: 27 March 2019

*Correspondence to:

Dr. Kalyani V. Pai Kakode, Email: kalyani.g.pai@ gmail.com

Copyright: (C) the author(s), publisher and licensee Medip Academy. This is an openaccess article distributed under the terms of the Creative Commons Attribution NonCommercial License, which permits unrestricted noncommercial use, distribution, and reproduction in any medium, provided the original work is properly cited.

\begin{abstract}
Background: To ascertain whether the pharmaceutical companies are following the WHO criteria for "Ethical medicinal drug promotion 1988" and "OPPI Code of pharmaceutical marketing practices, 2012" and to what extent. Also, to evaluate the therapeutic claims made by them in their drug promotional literature and other aspects.

Methods: Drug Promotional Literature (DPL) from different pharmaceutical companies was collected from various Medical practitioners and analysed to see if they achieved objectives. Therapeutic claims made by them were classified as authentic, exaggerated, controversial, false, and misinterpreted.

Results: Total 250 DPLs were collected and critically analysed for information content. Out of the $11 \mathrm{WHO}$ criteria for ethical medicinal drug promotion (1988), Majority $(30 \%)$ followed only $54.54 \%$ of the criteria while only $4 \%$ followed $100 \%$ of the criteria. The total number of claims in the 250 DPL were 354 of which $52.8 \%$ were authentic and $47.2 \%$ were misleading. From the misleading claims: $28.7 \%$ were exaggerated, $34.7 \%$ were controversial, $22.8 \%$ were false, $23 \%$ were ambiguous.

Conclusions: This study enabled us to find out to what extent the pharmaceutical industries follow the standard criteria for DPL and evaluate the claims made by them. DPL is one of the important sources of drug information. Some of the pharmaceutical companies failed to follow the WHO criteria while advertising their products in order to make them look more lucrative having an underlying commercial motive. As they influence prescribing patterns of the clinicians, misleading promotional literature would result in irrational prescribing of drugs. To avoid this, DPL has to be critically evaluated.
\end{abstract}

Keywords: Drug promotional literature, Ethical promotion

\section{INTRODUCTION}

Major breakthoughs in medical science have resulted in a substantial increase in the number of drugs entering the market. This adds up to already existing information reservoir.

The various sources of drug information are Scientific journals, textbooks, drug compendia, monograph, drug promotional literature, drug bulletins, websites, seminar, workshops, conferences and so on.
Pharmaceutical companies discover, develop, manufacture and market new drugs. ${ }^{2}$ They are interested in promoting the sale of new drugs manufactured by them by convincing physicians to prescribe their product. Different modes of drug promotion include visual aids, flip charts, leave-behinds, advertisements, gifts and audio-visuals. ${ }^{3}$ Drug companies spend huge amounts on sales promotion which includes cost of sales representatives, medical education programmes, advertisements, distribution of drug promotional literatures etc. ${ }^{4}$ 
Drug promotional literatures or DPLs are pamphlets or brochures printed by various pharmaceutical companies in order to promote the sale of products manufactured by them. They form the major marketing techniques. ${ }^{5}$

Due to the concise nature of DPLs, busy medical practitioners may sometimes rely on them as the primary source of drug information. They can be highly informative when it provides the authentic information in a nutshell as long as they have been critically analysed and reviewed if not, they can be misleading. ${ }^{5}$ Therefore, DPLs have to be critically analysed for their content to prevent irrational prescribing pattern. ${ }^{6}$

The ethical promotion of prescription medicines is vital to the pharmaceutical industry's mission of helping patients by discovering, developing and marketing new medicines. It helps to ensure that healthcare professionals have access to information they need, that patients have access to the medicines they need and that medicines are prescribed and used in a manner that provides the maximum healthcare benefit to patients. ${ }^{7}$ Pharmaceutical companies have to follow certain ethical guidelines, at the national and international levels, for drug promotional activities to ensure better healthcare through rational use of medicines. ${ }^{8}$

World Health Organization (WHO) ethical criteria for medicinal drug promotion defines promotion as "all informational and persuasive activities by manufacturers and distributors, the effect of which is to induce the prescription, supply, purchase, and/or use of medicinal drug". 9

The "World Health Organization (WHO) criteria for ethical medicinal drug promotion 1988" and "the Code of Pharmaceutical Marketing Practices of the International Federation of Pharmaceutical Manufacturers and Associations (IFPMA) 2012" are two guidelines at the international level. ${ }^{7,9}$ In India, drug promotion is largely governed by the "Organisation of Pharmaceutical Producers of India 2012(OPPI)". ${ }^{10}$ World Health Organization criteria for "Ethical medicinal drug promotion, 1988" is the backbone of self-regulatory code of OPPI and IFPMA which is supposed to regulate the promotional activity of pharmaceutical industries. ${ }^{5}$ All promotion making claims concerning medicinal drugs should be reliable, accurate, truthful, informative, balanced, up-to-date, and capable of substantiation and in good taste. ${ }^{9}$ Therefore, this study was carried out to critically analyse the information content of drug advertisements in drug promotional literature available to the Medical Practitioners.

\section{METHODS}

This was an observational, cross sectional study, carried out after taking permission from the Institutional Ethics Committee.
The drug promotional literatures (DPLs) in the form of pamphlets or brochures were collected from various medical practitioners, which were provided to them by medical representatives of various pharmaceutical companies.

A total of 250 DPLs were selected for analysis. DPLs promoting ayurvedic medicines, reminder advertisements, drug monograms, medical devices were excluded from the study.

\section{The drug promotional literatures were further categorised as follows}

- Vitamins and minerals

- Cardiovascular and renal system

- Central nervous system

- Respiratory system

- Endocrine system

- Gastrointestinal system

- Blood

- Anti-inflammatory

- Chemotherapy of microbial diseases

- Chemotherapy of neoplastic diseases

- Miscellaneous.

Analysed to see whether they follow "WHO criteria for ethical medicinal drug promotion 1988" and "OPPI Code of pharmaceutical marketing practices, 2012" and to what extent.

Critically evaluated for the therapeutic claims made by them with the help of currently available medical literature.

\section{The claims were further classified as follows ${ }^{5}$}

- $\quad$ Authentic: a claim found to be completely justified according to the reference or evidence quoted in support.

- Exaggerated: when a minor advantage of a drug was unnecessarily magnified showing exaggerated applications. When a claim extended beyond the actual benefit obtained by the patient following drug administration.

- False: when the claim in question was totally wrong. When there were no studies to support the use of the drug for a particular claim made in promotional literature.

- Controversial: when the claim in question was supported by some scientific evidence. However, contradictory reports were also found challenging the validity of the claim. When only a few clinical studies in standard literature supported claims made on promotional literature while others disposed them.

- Ambiguous: when a merit of a drug in a particular circumstance was extrapolated erroneously to other situations. Claim found to be vague in its description. 


\author{
References stated in the DPL were analysed for \\ retrievability and authenticity.
}

References searched on the internet for retrievability on Pubmed and Google. The retrievable references were also categorized as journal article, review article, metaanalysis, randomised control trials, preclinical studies, website, textbooks, data on file, prescribing information, purchase articles.

\section{Pictorial data in the DPLs were analysed to see the following}

\section{Type of picture}

- Whether related or unrelated to the drug, treatment or disease

- $\quad$ Presence of bar graphs, line graphs, pie charts, tables

This was a cross-sectional descriptive study. The statistical analysis for the study was carried out using frequency tables and percentages.

\section{RESULTS}

In this study 250 drug promotional literatures (DPLs) were used. These randomly collected DPLs were from 82 different pharmaceutical companies, both Indian and Multinational companies, all situated in different parts of India.

Majority of the DPLs were of chemotherapy of microbial disease $(16.4 \%)$, thus this being the most promoted pharmacological class of drugs. This was followed by antiinflammatory drugs (11.2\%), vitamins and minerals (10.8\%), endocrine system (10\%), blood $(9.2 \%)$, gastrointestinal system $(8.4 \%)$, miscellaneous $(8.4 \%)$, central nervous system $(8 \%)$, respiratory system $(7.6 \%)$, cardiovascular and renal system $(6.4 \%)$ and chemotherapy of neoplastic disease (3.6\%).

Each brochure was analysed according to the "WHO criteria for ethical medicinal drug promotion 1988" and "OPPI Code of pharmaceutical marketing practices 2012"

INN was mentioned by $248(99.2 \%)$ DPLs and all 250 $(100 \%)$ DPLs mentioned the brand name. Amount of active ingredients and approved therapeutic indications were mentioned by $224(89.6 \%)$ and $214(85.6 \%)$ respectively.

The name of excipient was the least mentioned criterion, only $20(8 \%)$ DPLs mentioned it. The dosage form or regimen was mentioned by $211(84.4 \%)$ DPLs, of which $201(80.4 \%)$ mentioned the dosage form while only 44 (17.6\%) mentioned the complete dosage regimen.

Criteria like side effects, precautions and contraindications and major interactions were mentioned in only one third of the DPLs, i.e. $82(32.8 \%), 83(33.2 \%)$ and $55(22 \%)$ respectively.

Name and address of the manufacturer was mentioned in $175(70 \%)$ of the DPLs, whereas 70 (28\%) DPLs mentioned only the company name without giving the complete address and 5 (2\%) DPLs neither gave company name nor address.

Total $202(80.8 \%)$ had cited references to support the claims and information mentioned in the DLPs while 48 $(19.2 \%)$ did not cite any reference. There was a total of 659 references out of which $498(75.5 \%)$ were retrievable and $161(24.5 \%)$ were not retrievable. From the retrieved references, $273(41.4 \%)$ were purchase articles wherein only abstracts were available.

The number of references cited in each DPL was noted. It ranged from a minimum of 1 reference to a maximum of 13 references per DPL. Most of the DPLs i.e. 80.2\% cited between 1 to 4 references. There was repetition of references in $8(3.2 \%)$ DPLs probably to increase the number of references given.

Four references were in languages other than English

The year of publication of the references was noted. The percentage of references within the last 10 years i.e. with the year of publication 2005 or later, was $60.80 \%$, and those with the year of publication before 2005 was $39.2 \%$.

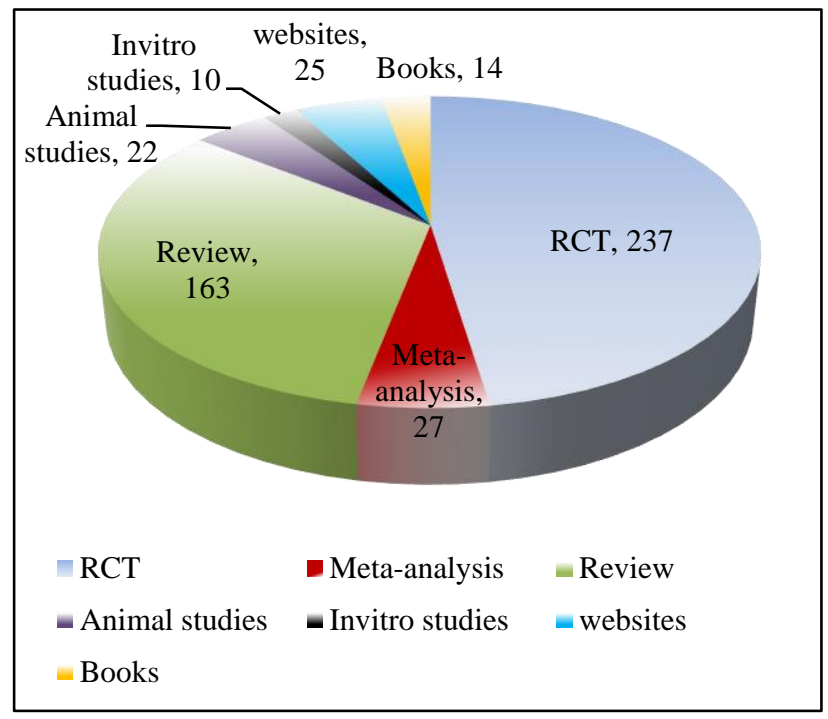

Figure 1: References cited in the DPLs (retrieved references).

\section{The extent of fulfilment of WHO criteria}

Majority i.e. 75 (30\%) DPLs fulfilled $54.54 \%$ of the WHO criteria, while very few i.e.10 (4\%) DPLs fulfilled $100 \%$ of the WHO criteria. 38 (15.2\%) DPLs fulfilled $63.63 \%$ of the WHO criteria and another $38(15.2 \%)$ DPLs fulfilled $90.90 \%$ of the WHO criteria. $36(14.4 \%)$ DPLs fulfilled 
$45.45 \%$ of the WHO criteria, 22(8.8\%) DPLs fulfilled $81.81 \%$ of the WHO criteria, $15(6 \%)$ DPLs fulfilled $72.72 \%$ WHO criteria, $12(4.8 \%)$ DPLs fulfilled $36.36 \%$ of the WHO criteria and 4 (1.6\%) DPLs followed $27.27 \%$ of the criteria.

In India, drug promotion is largely governed by the Organisation of Pharmaceutical Producers of India 2012 (OPPI) which is based on WHO criteria for ethical medicinal drug promotion 1988. As per OPPI, in addition to the WHO criteria the DPLs should also mention the date of production of the advertisement. Only 19 (7.6\%) out of 250 DPLs analysed, mentioned the date of production.

The WHO ethical criteria for medicinal drug promotion and The Code of Pharmaceutical Marketing Practices of the International Federation of Pharmaceutical Manufacturers and Associations (IFPMA) 2012 mention that words like "safe" and "no side effects" should generally be avoided and if used should be properly qualified. In this study, 10 (4\%) DPLs mentioned the drug as "safe" but it was not properly qualified.

The pictorial data in the DPLs was analysed and it was observed that in $123(49.2 \%)$ DPLs the illustrations were either related to the drug, treatment or the disease. On the other hand, pictorial data in 78 (31.2\%) DPLs was unrelated and $49(19.6 \%)$ DPLs did not have pictorial data at all. A total of 96 statistical figures were used in 60 (24\%) DPLs. Majority were bar graphs $(46.2 \%)$ followed by line graphs $(20.8 \%)$, tables $(19.7 \%)$, flow charts $(11.4 \%)$ and pie charts $(3.1 \%)$.

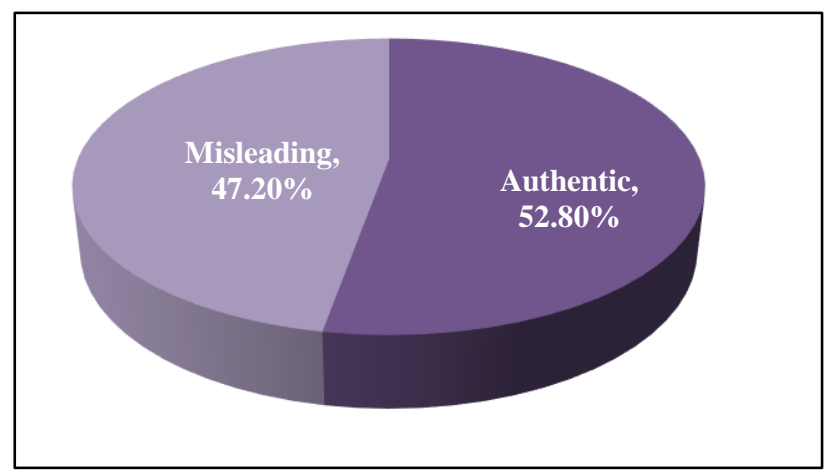

Figure 2: Percentage of authentic and misleading claims.

About 7 (2.8\%) DPLs had small print size, making the text too small to read, and leaving only the brand name legible.

Prices were mentioned in $15(6 \%)$ DPLs and cost comparison was given in $7(2.8 \%)$ of them.

Superlative claims like " No.1 brand ", "No1 prescribed SNRI", "Rank No.1", "Superior economy", "First of its kind", "Most economical", "Most widely prescribed", "First time in India" etc were used in 17 (6.8\%) DPLs.
Therapeutic claims made by the DPLs were analysed with the help of currently available medical literature like Goodman \& Gilman's. The pharmacological basis of therapeutics, Katzung's Basic and Clinical Pharmacology, Current Medical Diagnosis and Treatment and the Internet.

A total of 354 claims were made in the 250 DPLs, of which $187(52.8 \%)$ were authentic while 167 (47.2\%) were misleading. The misleading claims were again classified as exaggerated, false, controversial and ambiguous.

Controversial claims topped the list of misleading claims. There were $58(34.7 \%)$ controversial claims followed by $48(28.7 \%)$ exaggerated claims, $38(22.8 \%)$ false claims and $23(13.8 \%)$ ambiguous claims.

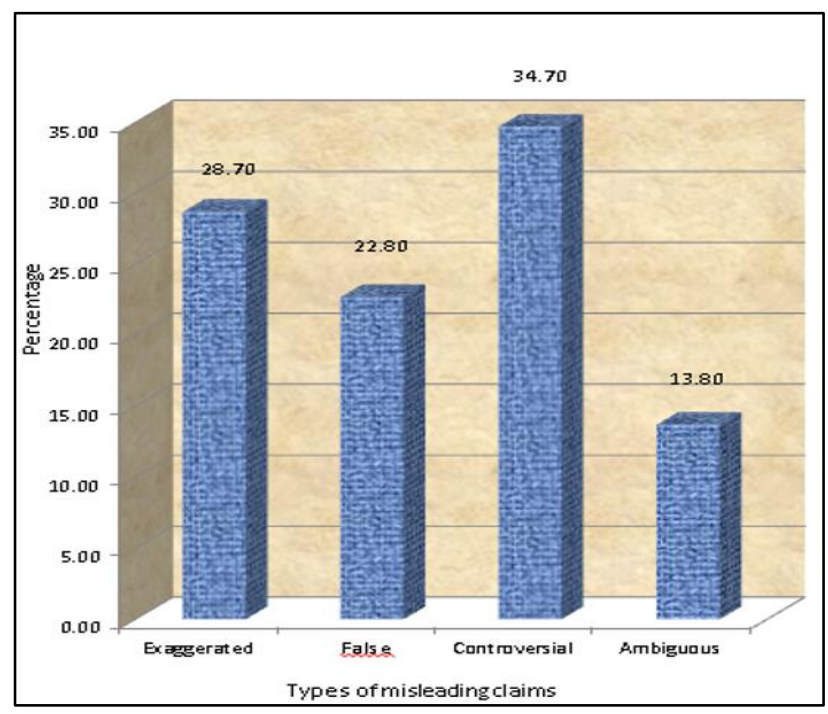

Figure 3: Percentage of each type of misleading claims.

\section{DISCUSSION}

Drug Promotional literature is one of the important sources of drug information. They can provide authentic information in brief and can be highly informative, provided they have been critically analysed and reviewed, otherwise they can be misleading. Because they influence the prescribing behavior of the medical practitioner, the DPLs have to be critically analysed for their content to prevent irrational prescribing pattern.

This study analysed 250 DPLs. The DPLs were categorized based on the pharmacological class of drugs. It was observed that drugs belonging to the class of chemotherapy of microbial disease, majority of which were antibiotics, were the most promoted ones $(16.4 \%)$. This observation is supported by a study by Mali SN et al, Rationality of drug promotional literature. $^{2}$

DPLs help in creating awareness about the existing as well as newer antibiotics available in the market. On the other hand, being most promoted, misuse of the antibiotics can 
result in the development of antibiotic resistance. Hence rational use of antibiotics is the need of the hour.

The next category which followed was anti-inflammatory drugs (11.2\%) and vitamins and minerals (10.8\%). Antiinflammatory drugs, especially nonsteroidal antiinflammatory drugs (NSAIDs) continue to be used very widely in the community. NSAIDs are used to temporarily relieve pain and inflammation. But overuse of NSAIDs for a long duration can cause adverse reactions especially in the gastrointestinal tract, kidney and liver.

The 250 DPLs consisted of 149 single drug formulations and 101 fixed dose combinations (FDCs) of which only 3 FDCs belonged to the $19^{\text {th }} \mathrm{WHO}$ list of essential medicines.

Each DPL was analysed according to the "WHO criteria for ethical medicinal drug promotion 1988" and "OPPI Code of pharmaceutical marketing practices 2012". .9,10

\section{International Nonproprietary Name (INN)}

International Nonproprietary Name (INN) identifies pharmaceutical substances or active pharmaceutical ingredients. The existence of an international nomenclature for pharmaceutical substances, in the form of INN, is important for the clear identification, safe prescription and dispensing of medicines to patients, and for communication and exchange of information among health professionals and scientists worldwide. Nonproprietary names are intended for use in pharmacopoeias, labelling, product information, advertising and other promotional material, drug regulation and scientific literature, and as a basis for product names, e.g. for generics.

Some countries have defined the minimum size of characters in which the generic nonproprietary name must be printed under the trade-mark labelling and advertising. In several countries the generic name must appear prominently in type at least half the size of that used for the proprietary or brand-name. ${ }^{11}$

\section{Brand name}

The brand name is the name given to a drug by the pharmaceutical company that manufactures it. On the basis of this study, it was observed that INN was mentioned by $248(99.2 \%)$ DPLs and all $250(100 \%)$ DPLs mentioned the brand name and in only $13(5.2 \%)$ DPLs, INN was at least half the size of brand name.

\section{Amount of active ingredients}

The elaboration of amount of active ingredients in each DPL helps the practitioner to prescribe the desired strength of the drug and also helps to titrate the dose as per the requirements. In some instances when a higher single dose is given or when an extended release formulation is prescribed, the dosing frequency is reduced which may help in better patient compliance. 224 (89.6\%) DPLs, among the ones analysed mentioned the amount of active ingredients.

\section{Name of excipients}

The FDA approves certain inactive ingredients that can be included in pharmaceutical products. However, not all inactive ingredients are always inactive. Alcohol is one of the examples. Also, patients may have allergic reactions or other adverse effects to the inactive ingredients. Hence it is mandatory to mention the excipient in the DPL. In this study it was found that the excipient was the least presented criterion with only 20 (8\%) DPLs mentioning it.

\section{Approved therapeutic indication}

The DPLs should mention the USFDA approved indications of the therapeutic drugs so as to promote rational prescribing patterns. The approved therapeutic indication was mentioned by 214 (85.6\%) DPLs.

\section{The dosage forms or regimen}

The dosage form or regimen was mentioned by $211(84.4 \%)$ DPLs, of which $201(80.4 \%)$ mentioned the dosage form while only $44(17.6 \%)$ mentioned the complete dosage regimen.

\section{Side effects}

Side effects were mentioned only by one third of the DPL i.e. $82(32.8 \%)$. Mention of the above two criteria i.e. dosage form or regimen and side effects, is important as regards to the compliance of a drug. A better side effect profile encourages better compliance by the patient. Among the dosage forms, it is seen that oral formulations are generally better accepted by the patients rather than parenteral formulations.

\section{Precautions and contraindications}

Infancy, childhood, pregnancy, lactation, old age, hepatic and renal diseases are special situations where dose adjustment becomes necessary for providing optimum therapeutic benefit. Thus, one of the important features of the WHO ethical criteria for medicinal drugs promotion is stating the precautions and contraindications which were mentioned in only one third of the DPLs, i.e. 83 (33.2\%).

\section{Major interactions}

When co-morbidities exist together there is a need of treating with a combination of drugs, therefore ruling out drug interaction is vital. Only 55 (22\%) DPLs mentioned the major interaction.

\section{Name and address of the manufacturer}

DPLs were categorized according to whether they belonged to Indian or Multinational Company (MNC). A 
majority of the companies were of Indian origin while the rest were MNC. Name and address of the manufacturer was mentioned in $175(70 \%)$ of the DPLs $70(28 \%)$ mentioned only the company's name without giving the complete address and $5(2 \%)$ neither gave company's name nor address. The MNC were found to follow the WHO criteria to a more extent than the Indian companies. This finding was similar to a study by Sarmila Nath and colleagues on "Promotional advertisements of drugs in a medical journal". 8

\section{References to scientific literature as appropriate}

The references given in support of claims are important to confirm the validity and authenticity of the DPLs. The references were classified depending on the source as Journal, website, books. Journal articles were subclassified as RCT's, meta-analysis, review and preclinical tests like animal or in-vitro studies.

\section{The extent of following WHO criteria}

Very few (4\%) pharmaceutical companies followed $100 \%$ of the WHO criteria for ethical medicinal drug promotion 1988, thus lacking in essential information regarding a drug.

\section{Date of production of advertisement}

The pharmaceutical companies are constantly striving to develop newer and better drugs. This would mean that new treatment options would be available to the patients. To promote their products the DPLs act as an important information source. To keep up with the changing trends in medical science, this source of information has to be up-todate and that is why DPLs have to mention the date of production of advertisements.

In India, drug promotion is largely governed by the Organisation of Pharmaceutical Producers of India 2012 (OPPI) which is based on WHO criteria for ethical medicinal drug promotion 1988. As per OPPI, in addition to the WHO criteria the DPLs should also mention the date of production of the advertisement. Only 19 (7.6\%) out of 250 DPLs analysed, mentioned the date of production.

\section{Pictorial data}

Promotional articles are usually made attractive using various illustrations or statistical figures to persuade doctors to prescribe the drugs promoted in them. The wording and illustrations in advertisements to physicians and related health professionals should be fully consistent with the approved scientific data sheet for the drug concerned or other source of information with similar content. ${ }^{9}$ It was seen that $31.2 \%$ of the pictures presented in DPLs were irrelevant. By occupying most of the area of the DPL, they reduced the space for providing vital information.
The statistical figures like bar graphs, pie charts, tables, flow charts, line graphs etc. arrange the data in an organized manner such that the reader can understand the information easily and quickly. Bar graphs are helpful to compare amounts and frequencies. Pie charts are used to show the relationship of a part to a whole wherein it displays data in sectors and usually represents percentage. Tables are excellent ways to display data in an organized way making interpretation easier. Flow charts are used to display sequence of activity, and line graphs are used for continuous variables.

\section{Safe / no side effects}

The WHO ethical criteria for medicinal drug promotion mentions that the word "safe" should only be used if properly qualified. ${ }^{9}$ According to The Code of Pharmaceutical Marketing Practices of the International Federation of Pharmaceutical Manufacturers and Associations (IFPMA) 2012, descriptions such as "safe" and "no side effects" should generally be avoided and should always be adequately qualified. ${ }^{7} 10$ (4\%) DPLs mentioned the drug as "safe" but it was not properly qualified.

\section{Legibility}

Sometimes more emphasis is placed on making the DPLs attractive with pictures and claims, in the process leaving very less area for the basic prescribing information. In order to include this information, the text was printed with a smaller font size, rendering it illegible. 7 (2.8\%) DPLs had small print size, making the text too small to read, and leaving only the brand name legible. The text should be fully legible. ${ }^{9}$

\section{Prices}

Different brands of the same drug have their own cost differences. It is not mandatory for the DPLs to mention prices, but it is beneficial for the practitioner to know the prices while prescribing the drugs to his patients. Mention of the prices in the DPL saves the practitioner's time from researching the same from other sources. Prices were mentioned in $15(6 \%)$ DPLs and cost comparison was given in $7(2.8 \%)$ of them.

\section{Superlative claims}

These overstated claims are unrelated to patient outcome, disease consequence, or the drug itself, but may have powerful influence on the medical practitioners prescribing pattern.

\section{Therapeutic claims}

WHO criteria for ethical medicinal drug promotion states that "All promotion-making claims concerning medicinal drugs should be reliable, accurate, truthful informative, balanced, up-to-date, capable of substantiation and in good 
taste. They should not contain misleading or unverifiable statements or omissions likely to induce medically unjustifiable drug use or to give rise to undue risks."

In this study therapeutic claims made by the DPLs were analysed with the help of currently available medical literature and classified as authentic and misleading. The misleading ones were sub classified as exaggerated, false, controversial and ambiguous.

Doctors may be influenced by extensive marketing and may hastily prescribe the new products without confirming the validity of the claims. This in turn will result in possible detrimental health related consequences like failure of treatment from the use of inappropriate drugs, undesirable adverse effects, rise in antibiotic-resistant microorganisms, and an increase in national health care expenditure.

Regional Ethics Committees have been set up at Mumbai, New Delhi, Chennai, and Chandigarh to collect complaints against unethical drug promotional advertisements which forward these complaints to the Drug Controller Authority to take necessary legal actions to discipline the erring pharmaceutical companies. Medical practitioners should play significant role to critically evaluate the information given in a DPL before taking it as a scientific source of information and forwarding more complaints about such defaulting companies to the Regulatory Authority. ${ }^{3}$

To ensure compliance by pharmaceutical companies with standard recommended guidelines for ethical promotion, a board should be formed with at least one member who is a trained pharmacologist and one representative from medical division of the company. ${ }^{7}$ In teaching hospitals, the department of pharmacology can undertake the task of analysing the promotional literature before the medical representatives present the literature to the medical practitioners.

Sessions on "Evaluation of Promotional Literature" should be conducted for interns and residents as they are the ones to interact with the pharmaceutical representatives. Also training in analysis of drug promotional literature should be imparted to undergraduate students to emphasize its importance. The ethical promotion of a drug and its rational prescription is possible with the combined efforts of the medical practitioner, pharmaceutical company and the regulatory bodies. These efforts will ensure in making the promotional literature not just a marketing strategy but also a useful, up-to-date, and accurate source of drug information.

Funding: No funding sources

Conflict of interest: None declared

Ethical approval: The study was approved by the Institutional Ethics Committee

\section{REFERENCES}

1. Shetty VV, Karve AV. Promotional literature: How do we critically appraise?. J Postgrad Med. 2008;54(3):217.

2. Mali SN, Dudhgaonkar S, Bachewar NP. Evaluation of rationality of promotional drug literature using World Health Organization guidelines. Indian J Pharmacol. 2010;42(5):267.

3. Khakhkhar T, Mehta M, Shah R, Sharma D. Evaluation of drug promotional literatures using WHO guidelines. J Pharmaceut Negative Results. 2013 Jan $1 ; 4(1): 33$.

4. Stimson GV. Do drug advertisements provide therapeutic information?. J Med Ethics. 1977;3(1):713.

5. Randhawa GK, Singh NR, Rai J, Kaur G, Kashyap R. A critical analysis of claims and their authenticity in Indian drug promotional advertisements. Adv Med. 2015;2015.

6. Rohra DK, Gilani AH, Memon IK, Perven G, Khan MT, Zafar H, Kumar R. Critical evaluation of the claims made by pharmaceutical companies in drug promotional material in Pakistan. J Pharm Pharm Sci. 2006;9(1):50-9.

7. IFPMA Code of Practice. Available at: http://www.ifpma.org/fileadmin /content/Ethics/IFPMA_Marketing_Code/The_Code/ IFPMA_Code_2006_Revision_EN.pdf.

8. Nath S, Bhowmick S, Dutta T, Chowrasia VR, Bhattacharya S, Chatterjee RN, Sarkar A, Mukherjee P. A study of promotional advertisements of drugs in a medical journal: an ethics perspective. Indian J Med Ethics. 2014 Oct;11(4).

9. Ethical criteria for medicinal drug promotion. World Health Organization.1988 May 13. Available at: http://www.who.int/medicinedocs/collect/ edmweb/pdf/whozip08e/whozip08e.pd.

10. OPPI Code of Pharmaceutical Practices. Mumbai: Organisation of Pharmaceutical Producers of India 2012. Available at: http:// www.ifpma. org/fileadmin/content/About\%20us/2\%20Members/A ssociations/Code

India/OPPI_Code_of_Pharmaceutical__Practices__2012.pdf.

11. Guidelines on the Use of INNs for Pharmaceutical Substances. $1997 . \quad$ Available at: http://www.who.int/medicines/services/inn/innguidan ce/en/.

Cite this article as: Kakode KVP, Bhandare PN. Critical analysis of drug promotional literature available to the medical practitioners. Int $\mathrm{J}$ Basic Clin Pharmacol 2019;8:918-24. 